\title{
Caracterización acústica de las róticas fricativas prepausales en español peninsular
}

\author{
Jordi Cicres y Beatriz Blecua \\ Universitat de Girona \\ jordi.cicres@udg.edu, beatriz.blecua@udg.edu
}

\begin{abstract}
Submitted: 10/07/2014. Accepted: 16/12/2014. Available on line: 11/12/2015
Citation / Cómo citar este artículo: Cicres, J. y Blecua B. Acoustic characterization of prepausal fricative rhotics in Peninsular Spanish . Loquens, 2(1), e019. doi: http://dx.doi.org/10.3989/loquens.2015.019

RESUMEN: El objetivo de este estudio es presentar una caracterización acústica de las consonantes róticas prepausales realizadas con un único componente fricativo en español peninsular. Para ello, se analizaron cuatro variables acústicas que dan cuenta de su estructura espectral (esto es, centro de gravedad, desviación estándar, asimetría y apuntamiento) y la duración. Los resultados del análisis se compararon estadísticamente con las características espectrales de las consonantes fricativas /s/ (con las que comparten punto de articulación) en la misma posición. Se han hallado diferencias estadísticamente significativas en las cinco variables, siendo el centro de gravedad en las fricativas superior en unos $2600 \mathrm{~Hz}$ al centro de gravedad de las róticas. Las fricativas alveolares también muestran un resultado superior en la desviación estándar y en la duración. Por el contrario, con respecto al apuntamiento y a la asimetría, los sonidos fricativos muestran valores inferiores. Finalmente, mediante el análisis lineal discriminante, se ha logrado un porcentaje de clasificaciones correctas superior al $94 \%$ teniendo en cuenta únicamente como variables discriminantes el centro de gravedad y la asimetría.
\end{abstract}

Palabras clave: róticas; fricativas; caracterización acústica; vibrantes.

ABSTRACT: Acoustic characterization of prepausal fricative rhotics in Peninsular Spanish.- The aim of this study is to present an acoustic characterization of the prepausal rothic consonants produced with a single fricative component in Peninsular Spanish. To this end, four acoustic variables that account for their spectral structure (that is, center of gravity, standard deviation, skewness and kurtosis) and duration were analyzed. Results were statistically compared to the spectral characteristics of fricative consonants /s/ (with which they share place of articulation) in the same position. The results show significant statistical differences among the five variables, being the center of gravity of the fricatives $2600 \mathrm{~Hz}$ higher than the center of gravity of the rhotics. Alveolar fricatives also show higher values for the standard deviation and for the duration. On the contrary, regarding kurtosis and skewness, fricative sounds show lower values. Finally, a correct classification rate over $94 \%$ was achieved through linear discriminant analysis taking into account only the center of gravity and skewness as discriminant variables.

Keywords: rothics; fricatives; acoustic characterization; vibrants.

\section{INTRODUCCIÓN}

Las consonantes róticas del español, a pesar de su gran variabilidad acústica y articulatoria, se agrupan tradicionalmente en dos categorías fonológicas: la rótica percusiva / $/$ / y la vibrante $/ \mathrm{r} /$, que solo se oponen en posición intervocálica (por ejemplo en el par mínimo carocarro). La percusiva aparece también en grupos consonánticos tras las consonantes $/ \mathrm{p} /, / \mathrm{t} /, / \mathrm{k} /, / \mathrm{b} /, / \mathrm{d} /, / \mathrm{g} / \mathrm{o} / \mathrm{f} /, \mathrm{y}$ enposiciónfinaldepalabraante unavocal $\left(\mathrm{v} \_\# \mathrm{v}\right.$, comoenes- tar aqui), mientras que la vibrante se encuentra en inicio de palabra o tras las consonantes $/ 1 /, / \mathrm{n} / \mathrm{o} / \mathrm{s} /$, como en $a l$ rededor, Enrique o Israel.

En posición implosiva, tanto ante consonante como ante pausa, la rótica no es contrastiva y pueden aparecer realizaciones de la percusiva y de la vibrante, con toda la variación que esto supone. En algunos casos, se atribuye la aparición de una u otra variante al énfasis con que se produce - «según un mayor o menor énfasis puede resultar simple, múltiple o fricativa» (Quilis, 1993, 
p. 332) - a la variante dialectal o a «circunstancias casi siempre estilísticas» (Martínez Celdrán, 1984, p. 337). Blecua (2001) considera que «en posición de coda aparece una vibrante simple, aunque puede convertirse en múltiple por un proceso de refuerzo» (p. 295; véase también Hualde, 2005, p. 182), y atribuye esta variación a la situación comunicativa. Asimismo, el locutor es otra fuente de variación (Blecua, Cicres y Gil, en prensa; García Antuña, 2012). En definitiva, parece que, en general, se trata de variación libre, y en cualquier caso no se especifica en qué circunstancias concretas predomina cada realización.

Desde el punto de vista fonético, la rótica percusiva se caracteriza como una breve fase de cierre situada entre dos elementos con mayor abertura (cf. Blecua, 2001, pp. 273-280; Cerdà, 1968, p. 23; Malmberg, 1965, p. 33; Martínez Celdrán y Rallo, 1995, p. 182; Navarro Tomás, 1918/1985, p. 117; Real Academia Española, 2011, p. 245; Walsh Dickey, 1997, p. 96). Este cierre no implica necesariamente contacto entre los articuladores, sino que puede tratarse de una constricción de mayor o menor grado. Acústicamente se refleja como una oclusión sonora, con componentes periódicos en frecuencias bajas, o bien puede presentar formantes o incluso fricción, si no llega a producirse el contacto apicoalveolar, como resulta frecuente en habla continua (cf. Blecua, 2001, 2006; Real Academia Española, 2011). La duración de esta fase se sitúa entre 20 y $22 \mathrm{~ms}$ (cf. como ejemplo Blecua, 2001; Lope Blanch, 1978; Martínez Celdrán y Rallo, 1995; Massone, 1988; Quilis, 1993; Romero, 2008), y es un rasgo característico de las róticas y relevante para interpretarlas como tales.

Los estudios que se ocupan de las róticas destacan la "posición intervocálica" de la percusiva. Cuando este sonido no se encuentra en contexto intervocálico, se observa un breve segmento con características vocálicas entre la consonante adyacente y la fase de cierre de la rótica. Este elemento aparecería tanto en posición posconsonántica (CrV) como preconsonántica (VrC) o prepausal, contexto que nos ocupa en el presente estudio, y se conoce por términos como "elemento esvarabático" (svarabhatkti; Lenz, 1892), "epentético", "parásito" o "vocal intrusiva" (Gili Gaya, 1921; Malmberg, 1965; Navarro Tomás, 1918/1985). Presenta una estructura formántica semejante a la vocal núcleo de la sílaba, y puede durar incluso más que la propia fase de oclusión (cf. Quilis 1993, pp. 337-342, para una descripción detallada de las características del elemento esvarabático).

En posición implosiva, por tanto, una de las realizaciones esperadas y que de hecho se considera como la más habitual (Bradley, en prensa; Hualde, 2005) es una breve fase de cierre seguida del elemento vocálico. Según Blecua (2001), esta sería la forma canónica en dicho contexto, aunque debido a procesos de relajación o refuerzo pueden aparecer también otras realizaciones sin elemento vocálico o con más de uno.

De hecho, en este contexto resultan también posibles las realizaciones sin elemento vocálico, es decir, formadas por un solo segmento que correspondería a la fase de cierre, y que puede ser una oclusión sonora, una aproximante o una fricción, así como casos de elisión. Todas estas manifestaciones se encuentran preferentemente en habla continua y, sobre todo, en conversación espontánea. Asimismo, se pueden encontrar róticas implosivas con más de una fase de cierre, que corresponderían a una realización vibrante. Aunque la articulación de la vibrante es distinta a la de la percusiva, tanto en el mecanismo de vibración como en el lugar de constricción (cf. Martínez Celdrán y Fernández Planas, 2007, pp. 144-156; Navarro Tomás, 1918/1985, p. 123; Real Academia Española, 2011, p. 249; Recasens, 1991; Recasens y Pallarés, 1999), acústicamente ambos sonidos están claramente relacionados, y se distinguen básicamente por el número de fases de cierre: una para la percusiva y dos o más en la vibrante, separadas por elementos vocálicos. Tal como indica Lindau (1985), «the taps look very much like the closure phase of a trill» (p. 166). Como en el caso de la percusiva, las fases de cierre de la vibrante pueden ser oclusivas o presentar formantes o fricción (cf. Blecua, 1999, 2001; Recasens, 1993, p. 76).

Blecua (2001) analiza de forma detallada la variación acústica en las róticas del español. Concretamente, en posición implosiva ante consonante describe siete posibles manifestaciones acústicas, según el número de componentes, el tipo de fase de cierre y la presencia o no de elemento vocálico (cf. también para este contexto Blecua, 2006, pp. 99-102, y Bradley, 2004, que incluye datos correspondientes a diferentes variedades dialectales). Como se puede comprobar, la variación en este contexto es muy elevada y no se han hallado factores contextuales que determinen claramente el tipo de realización. Cabe destacar que estos trabajos no consideran la posición prepausal, esto es, en final de grupo fónico, en que la variación posiblemente se vea incrementada, puesto que en esa posición siempre se produce un descenso en la intensidad y en la tensión articulatoria general. De hecho, en un estudio reciente (Blecua, Cicres y Gil, en prensa) se analizan las róticas prepausales para determinar la variación interlocutor y su posible rendimiento en la identificación de hablante, y se describen realizaciones no documentadas en los estudios anteriores, a las que se hará referencia de nuevo más adelante.

De todas las realizaciones posibles de la rótica, la fricativa es muy esporádica en español peninsular. ${ }^{1}$ Se describe en algunas variantes, en habla espontánea, como forma relajada de la vibrante /r/ (cf. Blecua, 2001; Martínez Celdrán y Fernández Planas, 2007, p. 159; Navarro Tomás, 1918/1985, p. 124; Quilis, 1993, p. 348; cf. Za-

En la bibliografía más tradicional, como Navarro Tomás (1918/1985), se denomina vibrantes simples fricativas a las realizaciones que más adelante se definen como aproximantes, que presentan formantes en la fase de cierre. Las variantes que se describen como asibiladas serían en ese caso las que presentan realmente fricción. 
hler y Daidone, 2014, para el habla de Málaga; Henriksen y Willis, 2010, para la variante de Jerez; Rodríguez Castellano, 1952, para el español de Asturias, entre otros), en general en posición intervocálica, pero también en grupos consonánticos en posición de ataque en zonas muy concretas: Navarro Tomás (1918/1985) describe la asibilación de la vibrante simple en este contexto, especialmente en el grupo $t r$, como «fenómeno dialectal corriente en parte de Álava, Navarra, Rioja y Aragón y muy extendido en la América de la lengua española» (p. 120). Según los resultados de Blecua (2001), también aparece de forma aislada (únicamente el $2 \%$ del total de casos analizados) como realización de la fase de cierre de la percusiva sin elemento vocálico, siempre ante consonante fricativa.

La Real Academia Española (2011) distingue las realizaciones asibiladas de las aproximantes:

No se trata, por tanto, de alófonos aproximantes, como la realización [I] o su variante con alargamiento [.ri], fruto del debilitamiento de [r] y [r], sino de realizaciones fricativas y tensas. El proceso de asibilación modifica el modo de articulación, que deja de ser rótico (percusivo o vibrante) para convertirse en fricativo. (p. 258)

En el espectrograma de una realización asibilada aparece fricción o turbulencia en la parte alta del espectro, lo que indica que se trata de una realización fricativa, y «si se compara [...] con el de una consonante fricativa, puede apreciarse que la fricción o turbulencia posee características semejantes en ambos tipos de sonidos» (Real Academia Española, 2011, p. 258). Según esta misma fuente, algunas realizaciones asibiladas se ensordecen, sobre todo en contacto con consonante sorda, pero buena parte de ellas conservan restos de sonoridad.

En las variantes del español de América es más frecuente la realización denominada fricativa o asibilada de la rótica, que puede aparecer en diferentes contextos según el dialecto (cf. Hualde, 2005, pp. 186-187; Quilis, 1993, 347-348; Quilis y Carril, 1971; Real Academia Española, 2011, pp. 260-262, o Morgan y Sessarego, en prensa, para un resumen de las variantes que incluyen alófonos fricativos o asibilados de las róticas y de los estudios existentes sobre estas realizaciones).

Sin embargo, en posición implosiva la variante fricativa está más limitada, especialmente ante consonante. En posición prepausal parece que está más extendida: «en muchas regiones de América Latina, la /r/ final de palabra se pronuncia como fricativa, pudiéndose ensordecer parcial o completamente» (Bradley, en prensa; véase también Real Academia Española, 2011, pp. 260-261). Ante consonante, en cambio, no es tan frecuente. Colantoni (2001), por ejemplo, no encuentra ninguna asibilación en coda en los dialectos de Argentina. En cambio, sí se documenta en la región central de Guatemala, aunque cada vez sea menos frecuente, así como en el altiplano de Ecuador: según Argüello (1978, citado en Bradley, 2004), en esta última región en habla descuidada pueden aparecer róticas fricativas en posición posnuclear ante consonantes coronales, ensordeciéndose si estas últimas son sordas, pero no se produce nunca fricción ante consonante heterorgánica.
Para comprobar estos datos, que no estaban demostrados acústicamente, Bradley (2004) realizó un estudio acústico detallado de los grupos /rC/ en el español del altiplano ecuatoriano, y confirma esta distribución: la realización asibilada puede aparecer ante consonante homorgánica (considerando como homorgánicas las coronales), contexto en que se encuentra en variación libre con [r], pero no se documenta ante otras consonantes, en que la rótica presenta elemento vocálico. Desde el punto de vista acústico, la descripción de rótica asibilada es «a period of strident frication, whose turbulence is indicated by the presence of aperiodic energy in the upper spectra» (Bradley, 2004, p. 202). La duración de este tipo de realización fricativa es de unos $50 \mathrm{~ms}$, que corresponde prácticamente a la suma de duraciones entre la fase de cierre y la vocal esvarabática de una realización no fricativa, lo que podría indicar cierto efecto de alargamiento compensatorio asociado con la asibilación, en ausencia del elemento esvarabático.

A pesar de que hasta el momento apenas se habían documentado róticas fricativas en coda silábica en español peninsular, en el estudio ya citado de Blecua, Cicres y Gil (en prensa) se obtuvieron ejemplos en posición prepausal con componentes fricativos. Para este experimento se siguió la metodología propuesta por Blecua (2001), de modo que se tuvieron en cuenta el número de componentes (cada una de las fases de abertura o cierre) y su naturaleza (elemento vocálico, oclusiva, fricativa o aproximante). La peculiaridad de este estudio es que analizaron grabaciones de habla espontánea, procedentes de diez locutores de español centropeninsular. En el ámbito descriptivo, se halló que predominaban claramente las realizaciones con uno o dos componentes (39\% y $34 \%$ del total, respectivamente), mientras que los casos de elisión representaban solo el $4 \%$ del total y los casos con tres o más componentes sumaban cerca de una cuarta parte $(24 \%)$.

Asimismo, se observó que cuando la rótica prepausal constaba de un único componente, este era mayoritariamente fricativo (65 \% de los casos analizados), algo que no se había obtenido en los estudios descriptivos anteriores basados en corpus de lectura, en que la incidencia de este tipo de realización era mínima. En cambio, el porcentaje de realizaciones con un primer componente fricativo (el que corresponde a la fase de cierre) era muy bajo en las realizaciones con dos o más componentes (Figura 1). Por otra parte, se apreció un aumento del porcentaje de fases de cierre aproximantes conforme aumentaba el número de componentes.

Pero lo más significativo del estudio es que se hallaron realizaciones fricativas en posiciones que no habían sido explicadas. Se trata de los casos de dos componentes en los que el segundo presenta fricción en lugar de una estructura formántica definida, característica del elemento vocálico, y que suponen el 57 \% de los casos (Figura 2). Las Figuras 3 y 4 muestran ejemplos de este tipo de realización con un primer componente oclusivo (Figura 3) o aproximante (Figura 4), y un segundo componente fricativo. 
Figura 1: Porcentaje de realizaciones aproximantes, fricativas u oclusivas del primer componente, según el número de componentes de la rótica en posición prepausal. Fuente: Blecua, Cicres y Gil (en prensa).

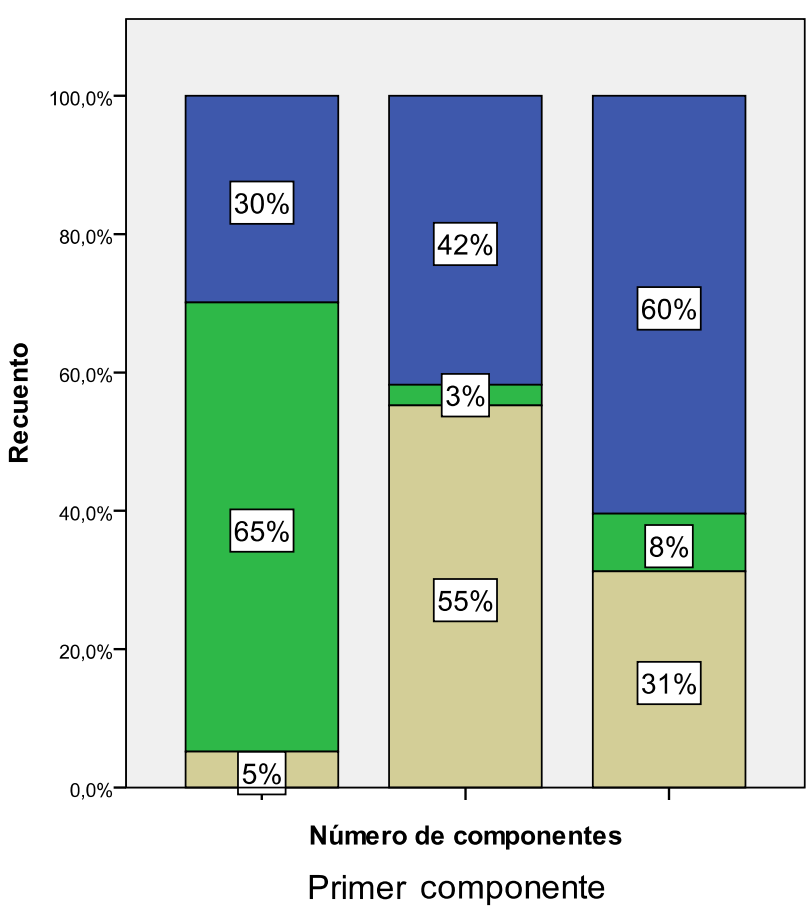

Aproximante $\square$ Fricativo $\square$ Oclusivo

Figura 2: Porcentaje de realizaciones del segundo componente de la rótica prepausal clasificado como elemento vocálico o fricción, según el número de componentes.

Fuente: Blecua, Cicres y Gil (en prensa).

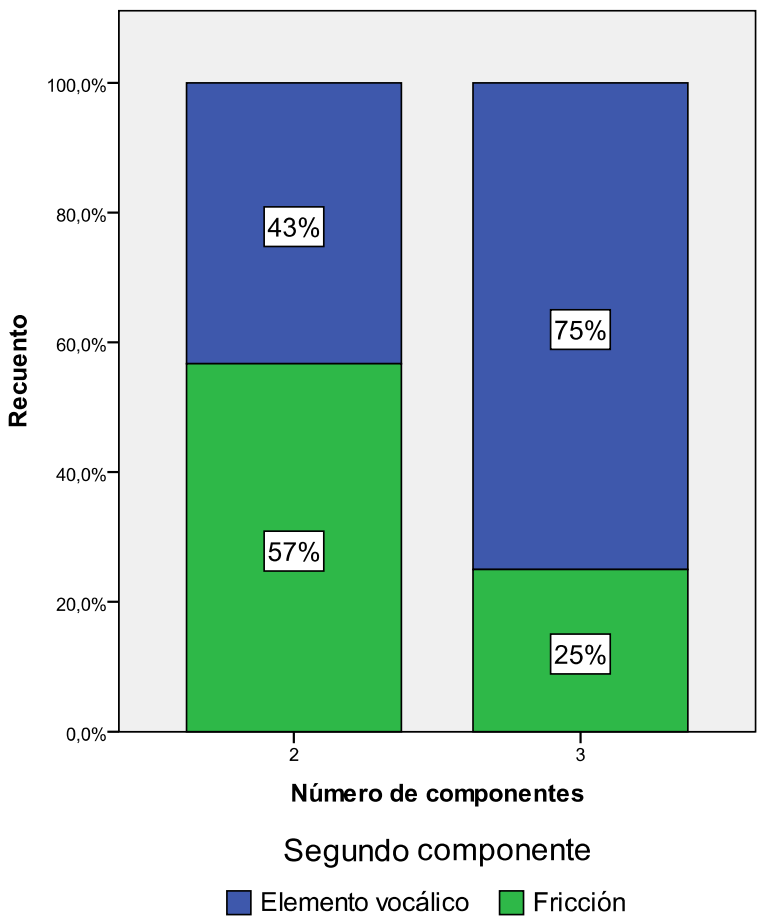

Figura 3: Espectrograma de la secuencia correr. Rótica final formada por dos componentes: oclusión y fricción. Fuente: Blecua, Cicres y Gil (en prensa).

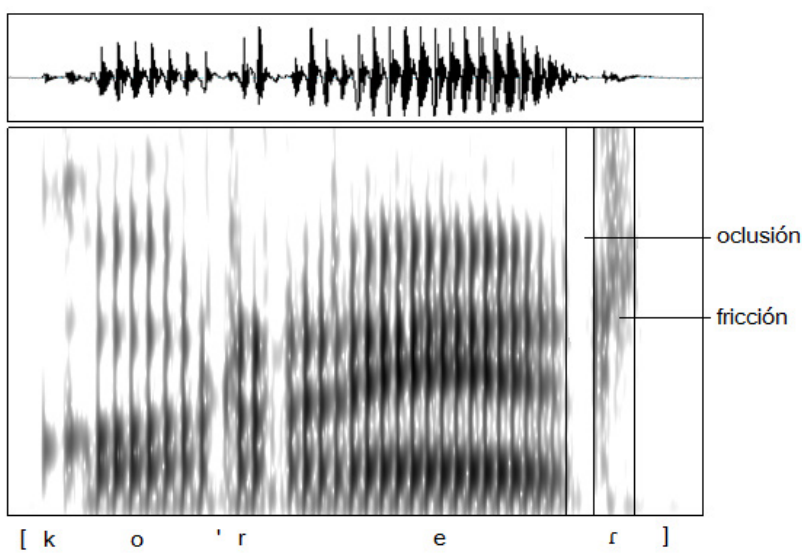

Figura 4: Espectrograma de la secuencia y ver. Rótica formada por dos componentes: aproximante y fricción. Fuente: Blecua, Cicres y Gil (en prensa).

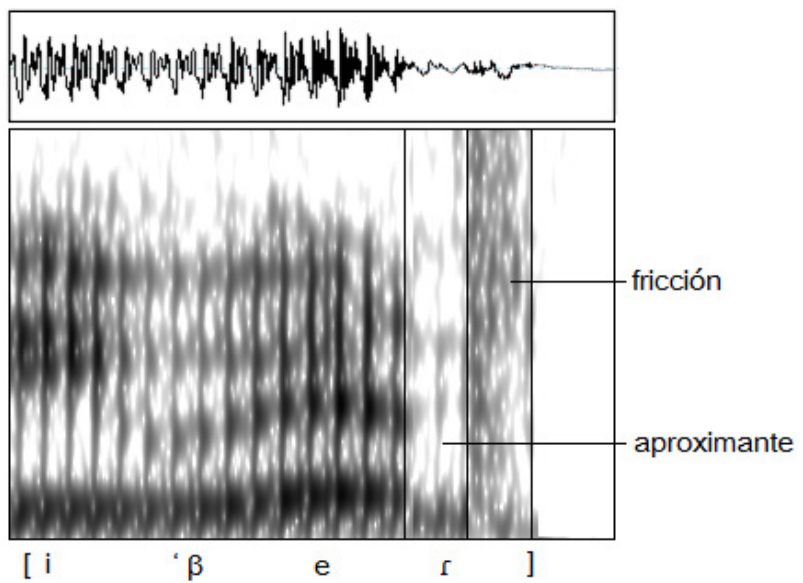

Estos casos resultan muy interesantes precisamente porque presentan fricción en el componente que correspondería al elemento esvarabático. Las diferentes explicaciones sobre la aparición de este segmento se basan siempre en sus características vocálicas, con una estructura muy similar a la de la vocal núcleo de sílaba. Si lo que aparece en la fase de abertura es una fricción, resulta difícil referirse a ella como elemento vocálico o vocal intrusiva. Tampoco tiene fácil explicación para la propuestas que, siguiendo el marco de la Fonología Articulatoria, consideran el elemento esvarabático como resultado de una falta de solapamiento articulatorio entre los gestos correspondientes a la / $/$ y la consonante siguiente, lo que hace que emerja (y se perciba) una porción de la vocal adyacente (cf. Bradley 2001, 2004; Romero, 2008). Bajo esta propuesta explican precisamente que en los ejemplos del español del altiplano de Ecuador no aparezca elemento esvarabático en los casos en que la realización es fricativa, ya que se solaparían los gestos de las dos consonantes. Asimismo, ejemplos como el de la Figura 4, con un 
primer componente aproximante, no responden a la estructura de fase de cierre + fase de abertura descrita en Blecua (2001), ya que en estos casos el segundo componente presenta un mayor grado de constricción articulatoria que el primero.

Los resultados también muestran que el porcentaje de realizaciones fricativas en el segundo componente (que correspondería a la fase de abertura) era mayoritario cuando la rótica tenía únicamente dos componentes, mientras que descendía hasta el $25 \%$ cuando tenía tres componentes (véase la Figura 2). ${ }^{2}$

Además, se encontraron algunos ejemplos esporádicos de róticas de dos componentes cuyo primer componente era fricativo y, el segundo, un elemento vocálico (Figura 5).

Figura 5: Espectrograma de la secuencia a ver. Rótica formada por dos componentes: fricción y elemento vocálico. Fuente: Blecua, Cicres y Gil (en prensa).

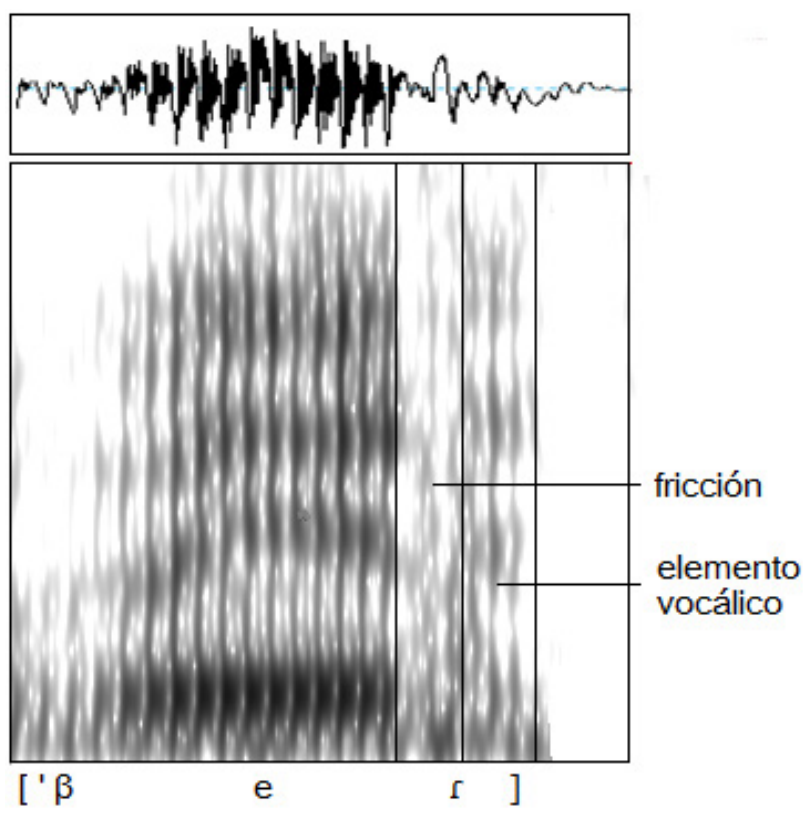

Sin embargo, los elementos que centran la atención del presente estudio son los sonidos róticos realizados mediante un único componente fricativo, cuyas características espectrales no se han descrito detalladamente hasta ahora para el español peninsular en este contexto.

\section{OBJETIVOS E HIPÓTESIS}

Este estudio se centra en el análisis de las características acústicas de las consonantes róticas en posición prepausal realizadas con un único componente fricativo en habla espontánea, con los siguientes dos objetivos:
- Caracterizar acústicamente las róticas prepausales realizadas como fricativas en español peninsular.

- Comparar las características espectrales de estas róticas con las fricativas alveolares también en posición prepausal con el fin de establecer si son segmentos similares 0 , por el contrario, difieren significativamente.

Para lograr el primer objetivo, se tendrán en cuenta las variables más ampliamente utilizadas en la descripción de la estructura espectral de los sonidos fricativos - como por ejemplo en Jongman, Wayland y Wong (2000), o Fu, Rodman, McAllister, Bitzer y Xu (1999) para el inglés, y Fernández, Feijóo, Balsa y Barros (1998) para el español- basadas en los cuatro primeros momentos espectrales: centro de gravedad, desviación estándar, asimetría y apuntamiento. Además, se tendrá en cuenta la duración del componente fricativo (véase § 3.2). Con el fin de alcanzar el segundo objetivo, se analizarán las características espectrales de fricativas alveolares en posición prepausal y se compararán estadísticamente con las róticas realizadas como fricativas, determinando qué variables permiten discriminar mejor $-\mathrm{y}$ en qué medida - a unos u otros segmentos.

En relación con este segundo objetivo, se plantean las siguientes hipótesis:

La estructura espectral de las róticas prepausales realizadas como fricativas diferirá significativamente de la de las realizaciones de /s/, puesto que el control articulatorio en las róticas presenta más dificultades que el de las fricativas, y por la variación en la combinación de componentes que se da en posición prepausal (Blecua, Cicres y Gil, en prensa).

En el caso de que la primera hipótesis sea validada, es consecuente plantear la siguiente:

Será posible identificar el vector de variables que mejor discrimina las róticas fricativas de las realizaciones del fonema /s/ en posición prepausal. En este sentido, se debería lograr un porcentaje alto de clasificaciones correctas de nuevos casos.

\section{METODOLOGÍA}

\subsection{Corpus}

El corpus está constituido por 10 grabaciones con una media de 29 minutos y medio de duración (y desviación estándar de 3 minutos y 4 segundos) correspondientes a habla espontánea masculina. Los locutores eran todos hombres con una edad comprendida entre los 25 y los 35 años, con formación universitaria y originarios de la zona centro de España (Madrid y Salamanca) sin ningún acento dialectal especialmente marcado. En ninguno de ellos se detectó ninguna patología ni rasgo fonético particular.

\footnotetext{
2 Bradley y Willis $(2012$, pp. 56, 58) describen un tipo de realización con un segundo componente fricativo, muy similar a las que aparecen en las Figuras 3 y 4, pero que corresponde a un alófono de la vibrante en posición intervocálica.
} 
Los locutores mantuvieron una conversación sobre temas cotidianos con un técnico del Laboratorio de Fonética del CSIC. Las grabaciones se realizaron utilizando canales separados para la voz del locutor (en una cabina insonorizada) y del técnico, de modo que no aparece ninguna interferencia o solapamiento entre las voces. Se utilizó un micrófono E6i Omnidireccional Earset. El formato de la grabación fue wav con una frecuencia de muestreo de $44100 \mathrm{~Hz}$ y resolución de 16 bits.

El número global de róticas prepausales con alguno de sus componentes fricativo es relativamente alto. En el corpus se encontraron 56 casos con el primer componente fricativo (que se corresponde con el 29,2 \% del total de róticas en posición prepausal del corpus) y 50 ejemplos con fricción en el segundo componente (que en este caso se corresponde con el 43,5\% del total de róticas ante pausa con dos o más componentes). Sin embargo, las róticas con un único componente fricativo suman solo un total de 48 realizaciones $(25 \%$ del total de róticas en posición prepausal) con una distribución entre los locutores muy irregular (Tabla 1). Destacan, en un extremo, los locutores 2 y 3 , con 10 y 19 casos respectivamente, y en el otro extremo el locutor 1, que no presenta ningún caso, y el resto de locutores, con un número de casos muy bajo (entre 1 y 5). Dadas las características de un corpus no preparado, basado en conversación, no se pudo controlar el número de casos para equilibrarlo ni la frecuencia de aparición de las realizaciones estudiadas, que es baja a pesar de contar con entrevistas de una duración considerable. Por otro lado, se encontró un número muy superior (414 casos) de fricativas /s/ prepausales, cuya distribución entre los locutores es más equilibrada.

Tabla 1: Número de róticas fricativas y fricativas alveolares prepausales, y su distribución entre los locutores del corpus.

\begin{tabular}{llllllllllll}
\hline & \multicolumn{10}{c}{ Locutor } & Total \\
\cline { 2 - 9 } & $\mathbf{1}$ & $\mathbf{2}$ & $\mathbf{3}$ & $\mathbf{4}$ & $\mathbf{5}$ & $\mathbf{6}$ & $\mathbf{7}$ & $\mathbf{8}$ & $\mathbf{9}$ & $\mathbf{1 0}$ & \\
\hline $\begin{array}{l}\text { Róticas } \\
\text { fricativas }\end{array}$ & 0 & 10 & 19 & 5 & 4 & 1 & 2 & 4 & 2 & 1 & 48 \\
$\begin{array}{l}\text { Fricativas } \\
\text { alveolares }\end{array}$ & 37 & 30 & 90 & 19 & 33 & 33 & 62 & 66 & 32 & 50 & 414 \\
\hline
\end{tabular}

\subsection{Análisis acústico}

Se delimitaron las róticas prepausales realizadas con un único componente fricativo, así como las fricativas alveolares prepausales, mediante la observación combinada de la onda sonora y del espectrograma. Para garantizar la fiabilidad del análisis, los dos autores segmentaron y categorizaron las róticas según sus características acústicas independientemente, alcanzando un porcentaje de coincidencia del $100 \%$. Los límites se marcaron en un TextGrid del programa de análisis acústico Praat (versión 5.3.45). La selección de las róticas se realizó después de haber analizado el número de componentes y la descripción de las características acústicas de todos los segmentos róti- cos prepausales. Se contemplaron cuatro clases de componentes distintos: elemento vocálico, oclusivo (con o sin barra de explosión), fricativo y aproximante. Los criterios que se siguieron para la determinación de las características de cada componente fueron los siguientes: presencia/ ausencia de formantes; presencia/ausencia de ruido en altas frecuencias; presencia/ausencia de período de oclusión (y ocasionalmente de barra de explosión), y número de zero-crossings por unidad de tiempo.

Sin embargo, la definición de cada componente no siempre es evidente, y la clasificación como fricción puede confundirse en algunos casos con la aproximante y, en otros, con la barra de explosión que en ocasiones aparece tras un periodo de oclusión. Así, se establecieron criterios objetivos para etiquetar correctamente los segmentos. En los ejemplos en los que aparecía energía dispersa en las frecuencias altas del espectrograma, la distinción entre aproximante y fricción se basó en la presencia observable de formantes, aunque fueran muy difusos (en cuyo caso el componente se etiquetaba como aproximante); en caso contrario, el componente se consideró fricativo. Para distinguir entre barras de explosión más largas de lo esperado y componentes fricativos se tuvieron en cuenta el número de zero-crossings (en caso de ser muy alto, el componente se consideró fricativo) y la duración de dicho fragmento (en caso de ser muy breve, se consideró que era la barra de explosión).

Para el análisis de su estructura espectral, se optó por calcular los momentos espectrales. Este tipo de análisis considera el espectro como una distribución de energía cuya forma puede ser caracterizada por su media, desviación estándar, asimetría y apuntamiento o curtosis. Siguiendo la metodología propuesta en Forrest, Weismer, Milenkovic y Dougall (1988), Zsiga (1993) y Jongman, Wayland y Wong (2000), se calcularon los momentos espectrales en el rango $0-10 \mathrm{kHz}$, a partir de un espectro FFT y con una potencia 2. Asimismo, para reducir los efectos de la coarticulación, se analizó la ventana correspondiente al $50 \%$ central de cada fricativa. El primer momento espectral (la media) indica en qué zona espectral se concentra la mayor parte de la energía. El segundo momento (la desviación estándar) mide la distancia de las frecuencias del espectro respecto del centro de gravedad, es decir, el grado de difusión de la energía en el espectro. El tercer momento espectral (el apuntamiento o curtosis) mide la forma del espectro, de modo que:

Positive kurtosis values indicate a relatively high peakedness (the higher the value, the more peaked the distribution), while negative values indicate a relatively flat distribution. Positive kurtosis thus suggests a clearly defined spectrum with well-resolved peaks, while negative kurtosis indicates a flat spectrum without clearly defined peaks. (Jongman, Wayland y Wong, 2000, p. 1253)

Finalmente, la asimetría se relaciona con la distribución de energía a lado y lado del centro de gravedad, es decir, a la inclinación de la forma del espectro, de modo que «positive skewness suggests a negative tilt with a concentration of energy in the lower frequencies. Negative skewness is associated with a positive tilt and a predo- 
minance of energy in the higher frequencies» (Jongman, Wayland y Wong, 2000, p. 1253).

\subsection{Análisis estadísticos}

En relación con el primer objetivo (caracterizar acústicamente las róticas prepausales realizadas como fricativas), se utilizó una combinación de datos descriptivos (media y desviación estándar de las cinco variables incluidas en el análisis) y pruebas estadísticas (mediante SPSS, versión 19) con el fin de medir el posible efecto de la vocal precedente y del locutor. Para ello, se llevaron a cabo dos análisis de efectos mixtos lineales (utilizando el contexto vocálico anterior como variable fija y el locutor como variable aleatoria, para estimar el efecto de la vocal precedente, y a la inversa para estimar el efecto del locutor), que se prefirieron a las pruebas ANOVA al poder incluir en el análisis el efecto de la variación entre locutores.

En relación con el segundo objetivo (comparar las características espectrales de estas róticas con las fricativas alveolares también en posición prepausal), se partió de las dos hipótesis planteadas. La primera hipótesis predice que la estructura espectral las róticas fricativas diferirá significativamente de la de las fricativas alveolares. Para contrastar esta hipótesis se realizaron también análisis de efectos mixtos lineales con el fin de comparar las características espectrales de las róticas fricativas con los sonidos fricativos prepausales, que en esta posición son siempre alveolares y sordos. En estos test se utilizaron los cuatro primeros momentos espectrales y la duración de los segmentos analizados como variables dependientes, el segmento (rótico vs. fricativo) como efecto fijo y el locutor como efecto aleatorio.

Para contrastar la segunda hipótesis, se realizó un análisis lineal discriminante mediante el método de inclusión por pasos (utilizando el estadístico lambda de Wilks con criterios de entrada de 3,84 y de salida de 2,71 para el valor $F$ ). Con el fin de comprobar el potencial discriminante, se utilizó el método de clasificación dejando uno fuera. Este método consiste en un sistema de validación cruzada que permite evaluar la capacidad predictiva de la función discriminante.

\section{RESULTADOS}

La presentación de los resultados se organiza en dos apartados. En primer lugar, se describen las características acústicas de las róticas fricativas prepausales analizadas, y a continuación se comparan con las realizaciones de la consonante fricativa alveolar /s/ en la misma posición.

\subsection{Caracterización acústica de las róticas fricativas prepausales}

Las Figuras 6 y 7 muestran el espectrograma (junto con el oscilograma; Figura 6) y el espectro (correspondiente a la parte central del segmento fricativo; Figura 7) de una rótica en posición prepausal clasificada como fricativa, como ejemplo del tipo de realización objeto de estudio. Se trata de una realización con un solo componente, en la que no aparece elemento vocálico, sino una sola fase de cierre. En el espectrograma se puede apreciar la presencia de energía en las frecuencias altas en el segmento rótico, así como la ausencia de formantes. En este caso se trata de una realización ensordecida, sin rastro de sonoridad. ${ }^{3}$

Figura 6: Espectrograma de la secuencia (agrad)ecer (locutor 6). Se marca entre líneas verticales el segmento correspondiente a la rótica fricativa.
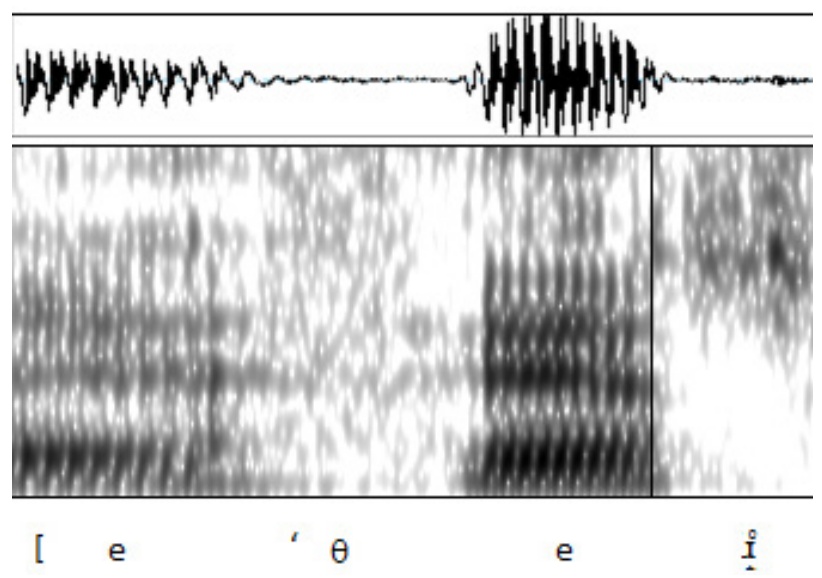

Figura 7: Espectro del centro de la rótica fricativa correspondiente a la consonante final de la secuencia agradecer (locutor 6).

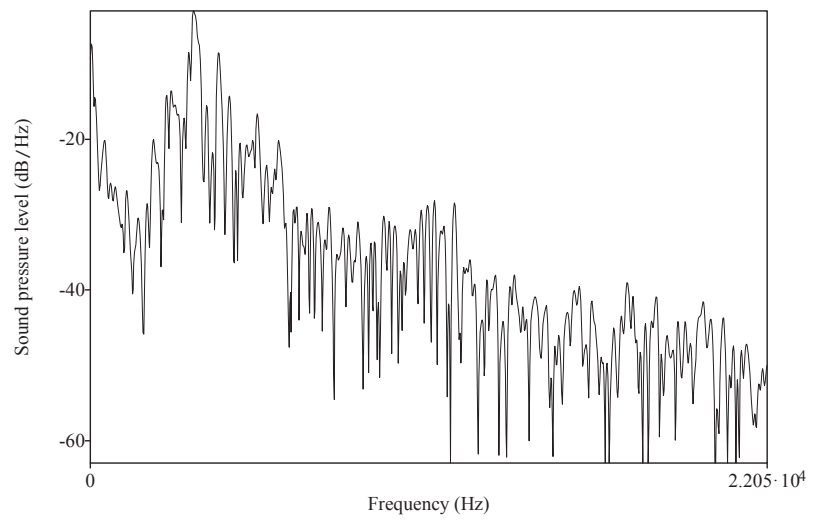

\footnotetext{
3 Para simplificar y no entrar en disquisiciones sobre la transcripción fonética más adecuada para cada realización, lo cual no es el objetivo de este trabajo, se han utilizado los símbolos fonéticos [II] y [II] para la rótica fricativa sonora y la ensordecida respectivamente, siguiendo la notación que se utiliza en Blecua (2001) o Bradley y Willis (2012; cf. Blecua, 2001, pp. 15-22, para una propuesta de transcripción de las diferentes variantes).
} 
La Tabla 2 recoge los valores medios de todas las variables tras el análisis conjunto de los datos.

Tabla 2: Valores medios de las róticas fricativas prepausales (entre paréntesis se muestra la desviación típica).

\begin{tabular}{ccccc}
\hline $\begin{array}{c}\text { Centro de } \\
\text { gravedad } \\
(\mathbf{H z})\end{array}$ & $\begin{array}{c}\text { Desviación } \\
\text { estándar } \\
\mathbf{( H z )}\end{array}$ & $\begin{array}{c}\text { Apunta- } \\
\text { miento } \\
\mathbf{( H z )}\end{array}$ & $\begin{array}{c}\text { Asimetría } \\
\mathbf{( H z )}\end{array}$ & $\begin{array}{c}\text { Duración } \\
(\mathbf{s})\end{array}$ \\
\hline 1127,7 & 1193,9 & 18,54 & 2,63 & 0,08 \\
$(647)$ & $(485,4)$ & $(26,12)$ & $(2,01)$ & $(0,03)$ \\
\hline
\end{tabular}

Un aspecto destacable es la duración de las róticas realizadas como fricativas, con una media de $80 \mathrm{~ms}$. Si se compara con los valores medios de la fase de cierre de una percusiva, 20-22 ms (véase la Sección 1), prácticamente cuadruplica su duración, e incluso si se tiene en cuenta también el elemento vocálico (es decir, sumando la fase de cierre y la fase de abertura), las róticas fricativas superan ampliamente la duración total de las realizaciones de dos componentes (cf. Bradley, 2004, p. 204, que también describe duraciones más elevadas en las róticas fricativas, aunque menores que aquí). Hay que tener en cuenta, sin embargo, que la desviación típica es elevada, ya que entre el valor mínimo $(32 \mathrm{~ms})$ y el máximo $(153 \mathrm{~ms})$ la distancia es muy relevante.

Volviendo a la Tabla 2, se puede observar que la desviación estándar en todos los parámetros es alta. Esta variación se podría explicar bien por el efecto del contexto (en este caso, la vocal precedente), bien por el efecto del locutor. Mediante un análisis de efectos mixtos lineales (con el contexto anterior como efecto fijo y el locutor como efecto aleatorio) comprobamos que la incidencia de la vocal anterior no explica la variación observada, puesto que los resultados aportan valores no significativos en todos los casos, excepto en la duración (Tabla 3). Hay que señalar que la distribución de los datos es homogénea en todas las combinaciones de variables (estadístico de Levene $>, 05)$.

Tabla 3: Resultados de los análisis de efectos mixtos lineales (con el contexto anterior como efecto fijo y el locutor como efecto aleatorio).

\begin{tabular}{lcc}
\hline & $\boldsymbol{F}$ & $\boldsymbol{p}$ \\
\hline Centro de gravedad & 0,354 &, 839 \\
Desviación estándar & 0,127 &, 972 \\
Apuntamiento & 0,667 &, 619 \\
Asimetría & 0,768 &, 553 \\
Duración & 2,848 &, 036 \\
\hline
\end{tabular}

Sin embargo, tomando en consideración el locutor como efecto fijo (y la vocal anterior como efecto aleatorio), observamos que el análisis de efectos mixtos lineales evidencia diferencias significativas para todas las variables consideradas (Tabla 4). Estos resultados son coherentes con los hallados en estudios centrados en el análisis de los segmentos fricativos desde la óptica de la fonética forense (o judicial), como por ejemplo en Cicres (2011). En esta línea, se aportan nuevas evidencias de que los sonidos fricativos (tanto si se trata de fonemas fricativos como si son componentes fricativos correspondientes a otros fonemas) presentan un alto grado de variación inter-locutor.

Tabla 4: Resultados de los análisis de efectos mixtos lineales (con el locutor como efecto fijo y la vocal anterior como efecto aleatorio).

\begin{tabular}{lcc}
\hline & $\boldsymbol{F}$ & $\boldsymbol{p}$ \\
\hline Centro de gravedad & 4,536 &, 001 \\
Desviación estándar & 2,808 &, 015 \\
Apuntamiento & 9,632 & $<, 001$ \\
Asimetría & 4,679 & $<, 001$ \\
Duración & 3,736 &, 003 \\
\hline
\end{tabular}

De hecho, al comparar los espectrogramas y espectros de diferentes locutores, como los presentados en las Figuras 6 y 7 (correspondientes al locutor 6 del corpus) y 8 y 9 (del locutor 4 ), se observan diferencias claras tanto en la duración como en la forma del espectro. Por un lado, la duración de la rótica del locutor 6 (77 ms) es un $63 \%$ superior a la del locutor 4 (47 ms); por el otro, el centro de gravedad es también más alto en el locutor $6(3227 \mathrm{~Hz})$ que en el locutor $4(786 \mathrm{~Hz})$. Sin embargo, los datos correspondientes a la desviación estándar $(1249 \mathrm{~Hz}$ para el locutor 6 vs. $2252 \mathrm{~Hz}$ para el locutor 4), asimetría $(0,89$ vs. 4,66 Hz) y apuntamiento $(17,79$ vs. $27,05 \mathrm{~Hz})$ son claramente superiores en el locutor 4.

Figura 8. Espectrograma de la secuencia salir (locutor 4). Se marca entre líneas verticales el segmento correspondiente a la rótica fricativa.

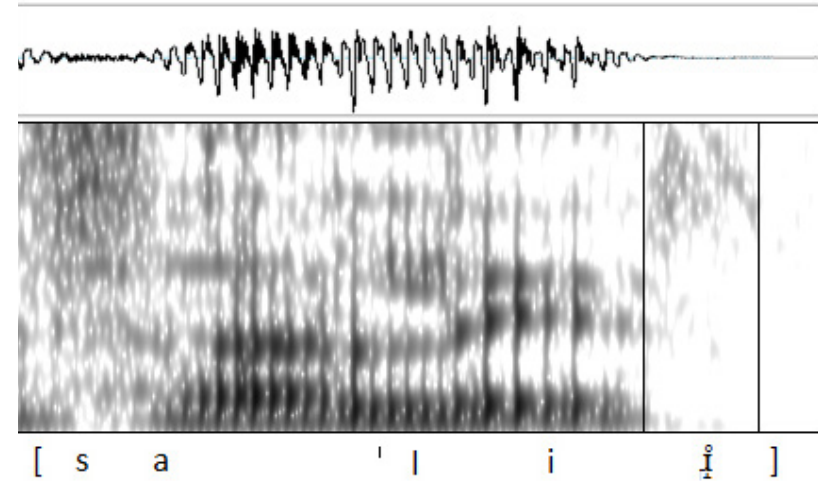


Figura 9: Espectro del centro de la rótica fricativa correspondiente a la consonante final de la secuencia salir (locutor 4).

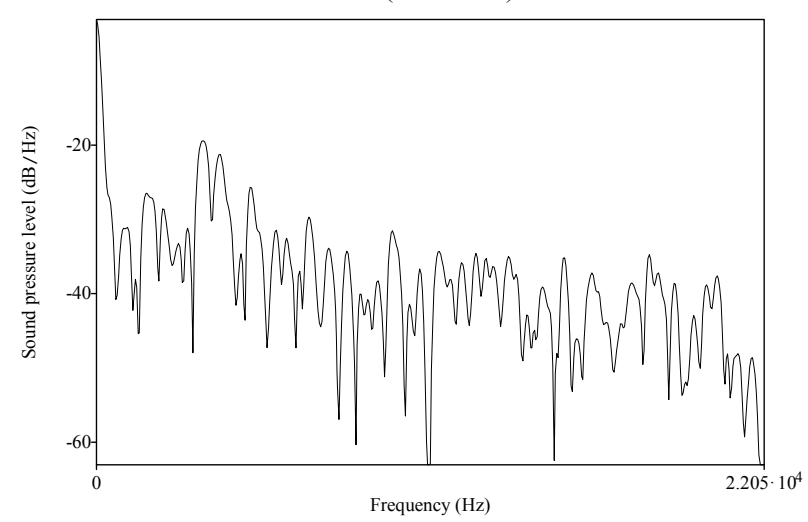

\subsection{Comparación entre róticas fricativas y fricativas alveolares prepausales}

El segundo de los objetivos planteados persigue determinar si las características espectrales de la róticas prepausales analizadas son similares a las fricativas alveolares "canónicas" también en posición prepausal. La Figura 10 muestra el espectrograma y el oscilograma de una fricativa alveolar prepausal, mientras que la Figura 11 muestra el espectro de la misma.

Figura 10: Espectrograma de la secuencia inglés. Se marca entre líneas verticales el segmento correspondiente a la fricativa alveolar

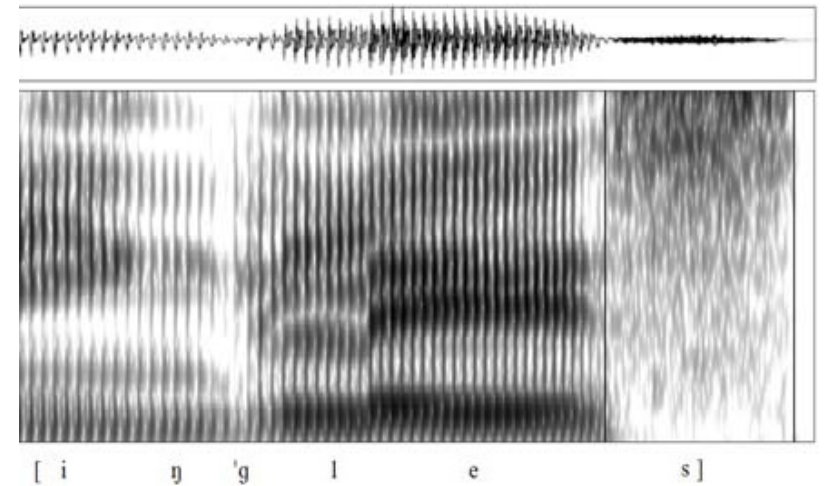

Para ello, en primer lugar obtuvimos los datos descriptivos (Tabla 5) de las mismas variables analizadas en los componentes fricativos de las róticas prepausales (en la Tabla 2). Observamos que el centro de gravedad en las fricativas es superior en unos $2600 \mathrm{~Hz}$ al centro de gravedad de las róticas. Las fricativas alveolares también muestran un resultado superior en la desviación estándar y en la duración. En cambio, para las variables referentes al apuntamiento y a la asimetría, los sonidos fricativos muestran valores inferiores.
Figura 11. Espectro del centro de la fricativa alveolar correspondiente a la consonante final de la secuencia inglés.

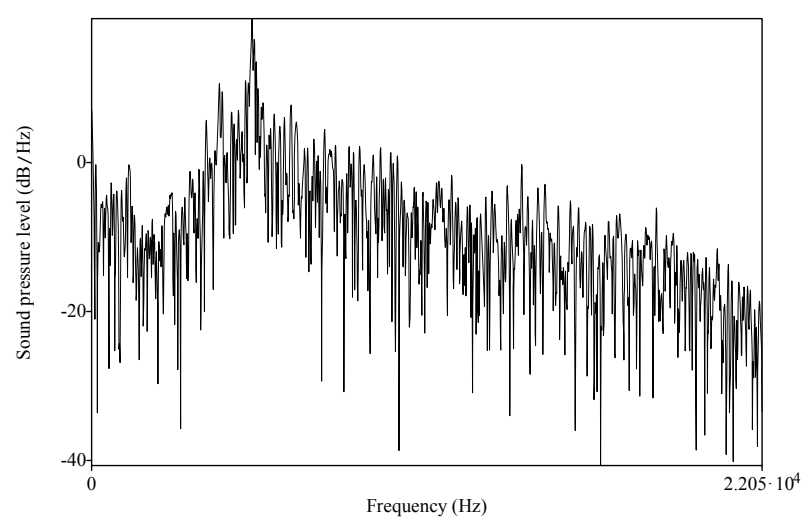

Tabla 5: Valores medios del conjunto de fricativas prepausales (entre paréntesis se muestra la desviación típica).

\begin{tabular}{ccccc}
\hline $\begin{array}{c}\text { Centro de } \\
\text { gravedad } \\
(\mathbf{H z})\end{array}$ & $\begin{array}{c}\text { Desviación } \\
\text { estándar } \\
\mathbf{( H z )}\end{array}$ & $\begin{array}{c}\text { Apunta- } \\
\text { miento } \\
\mathbf{( H z )}\end{array}$ & $\begin{array}{c}\text { Asimetría } \\
\mathbf{( H z )}\end{array}$ & $\begin{array}{c}\text { Duración } \\
\mathbf{( s )}\end{array}$ \\
\hline 3847,4 & 1798,6 & 2,71 & 0,5 & 0,12 \\
$(1120,9)$ & $(438,9)$ & $(4,95)$ & $(0,73)$ & $(0,05)$ \\
\hline
\end{tabular}

Las Figuras 12, 13 y 14 muestran los diagramas de caja de las variables analizadas. Se observa que existen diferencias evidentes tanto en la distribución como en los cuartiles entre las róticas realizadas como fricativas y las fricativas alveolares. El análisis de efectos mixtos lineales, comparando las cinco variables de análisis en los componentes fricativos de las róticas y las fricativas prepausales (y utilizando como efectos aleatorios el locutor y la vocal anterior) han resultado altamente significativas ( $p$ $<, 001$ en todos los casos), lo que demuestra que acústicamente se trata de fricciones bien diferenciadas. Así, se valida la primera hipótesis.

Para contrastar la segunda hipótesis, que prevé que se podrá diferenciar eficazmente entre róticas fricativas y fricativas "canónicas" mediante un vector de variables, se realizó un Análisis Lineal Discriminante (ALD).

Las variables relevantes para la discriminación entre los grupos han sido el centro de gravedad y la asimetría. Por el contrario, el apuntamiento, la desviación estándar y la duración no se han empleado en el ALD. La Tabla 6 muestra la matriz de estructura (correlaciones intra-grupo combinadas entre las variables discriminantes y las funciones discriminantes canónicas tipificadas) y, la Tabla 7, las funciones en los centroides de los grupos (funciones discriminantes canónicas no tipificadas evaluadas en las medias de los grupos). Se observa que el grupo formado por las róticas fricativas toma un valor negativo en la función discriminante (siendo, pues, significativamente menores los valores de centro de gravedad, desviación estándar y duración, y mayores los valores de asimetría y apuntamiento). 
Figura 12: Diagramas de caja correspondientes al centro de gravedad y desviación estándar según el tipo de segmento analizado.

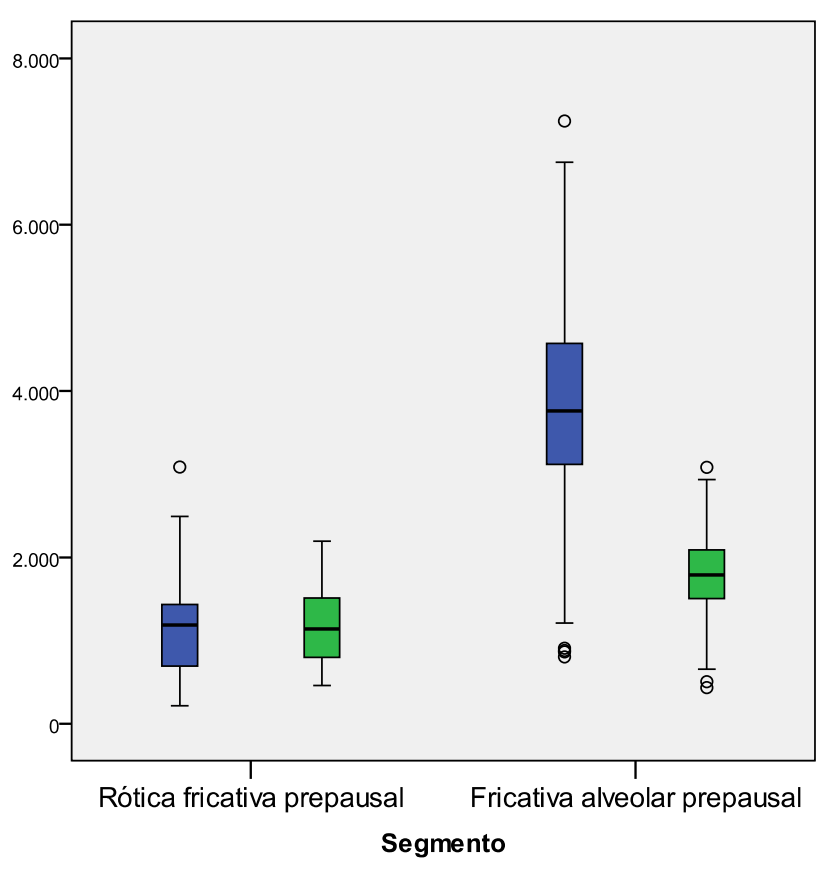

Centro de gravedad

Desviación estándar

Figura 13: Diagramas de caja correspondientes al apuntamiento y asimetría según el tipo de segmento analizado.

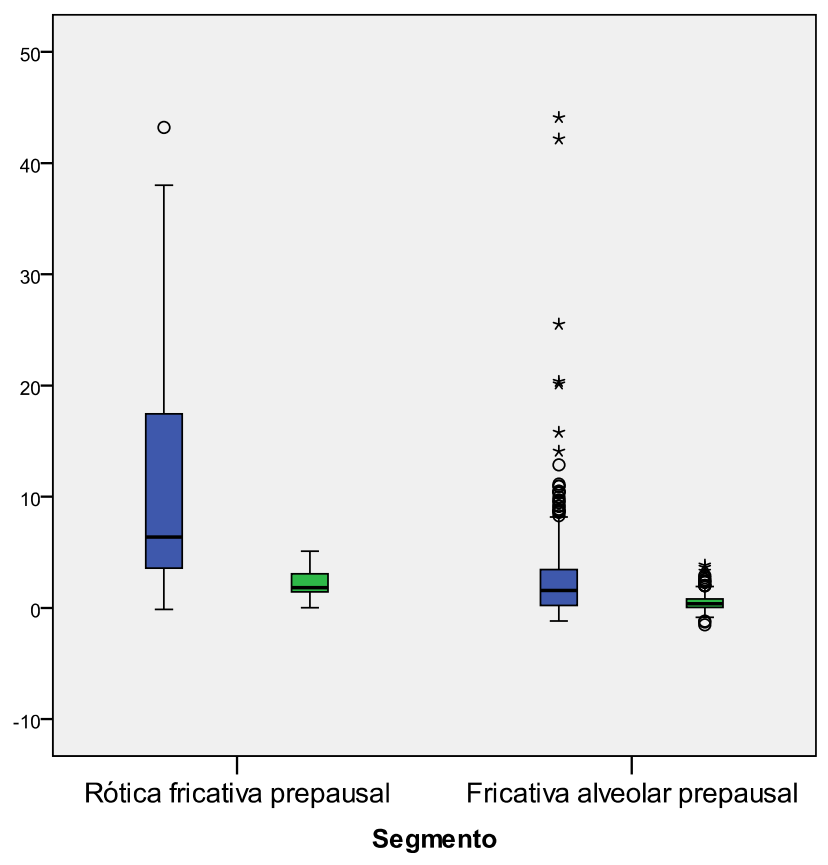

Apuntamiento $\square$ Asimetría
Figura 14: Diagramas de caja correspondientes a la duración según el tipo de segmento analizado.

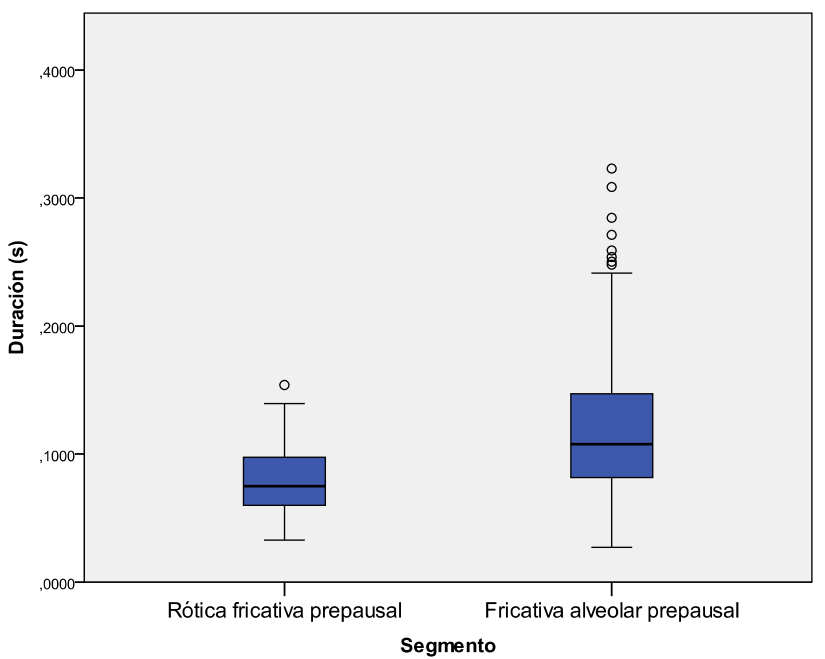

Tabla 6: Matriz de estructura para el análisis lineal discriminante.

\begin{tabular}{lc}
\hline & Función \\
\cline { 2 - 2 } Centro de gravedad & $\mathbf{1}$ \\
Asimetría &, 866 \\
Apuntamiento $^{\mathrm{a}}$ &,- 786 \\
Desviación estándar $^{\mathrm{a}}$ &,- 541 \\
Duración (s) $^{\mathrm{a}}$ &, 408 \\
\hline
\end{tabular}

a. Esta variable no se emplea en el análisis.

Tabla 7: Funciones en los centroides de los grupos para el análisis lineal discriminante.

\begin{tabular}{lc}
\hline \multicolumn{1}{c}{ Segmento } & Función \\
\cline { 2 - 2 } & $\mathbf{1}$ \\
\hline Rótica fricativa prepausal & $-2,589$ \\
Fricativa alveolar prepausal &, 300 \\
\hline
\end{tabular}

Por otro lado, el ALD logra clasificar correctamente el $94,8 \%$ de los casos en la clasificación de los casos originales y el 94,6 \% en la validación cruzada.

\section{DISCUSIÓN Y CONCLUSIONES}

El estudio, centrado en el habla espontánea, ha descrito desde el punto de vista acústico cómo son las róticas fricativas prepausales de un único componente, que suponen un porcentaje nada desestimable del total de róticas prepausales. A grandes rasgos, la fricción se caracteriza por un centro de gravedad bajo $(1127,7 \mathrm{~Hz}$ de media) y un alto apuntamiento $(18,54 \mathrm{~Hz})$, así como por una dura- 
ción considerable ( $80 \mathrm{~ms}$ de media). Sin embargo, hay que destacar también que, para todos los parámetros, la variación encontrada ha sido elevada, y se ha atribuido al efecto de los locutores, puesto que el contexto previo (el timbre de la vocal anterior) no se descubrió relevante.

En segundo lugar, el estudio ha puesto de manifiesto que las róticas fricativas prepausales, aun presentando todos los rasgos característicos de los sonidos correspondientes a fonemas fricativos, es decir, un aumento rápido de zero-crossings en relación con los sonidos colindantes y ruido turbulento (Barreiro Bilbao, 1999-2000; Martínez Celdrán y Fernández Planas, 2007; Quilis, 1993, por citar solo algunas obras centradas en el español), presentan diferencias significativas en comparación con las realizaciones del fonema/s/ (con el que comparten punto de articulación) que pueden aparecer en la misma posición (prepausal). Así, los sonidos fricativos que representan el fonema /s/ tienen un centro de gravedad y una desviación estándar significativamente más altos (alrededor de los $3850 \mathrm{~Hz}$ y $1800 \mathrm{~Hz}$ respectivamente para /s/, frente a $1140 \mathrm{~Hz}$ y $1194 \mathrm{~Hz}$ para la rótica), una duración mayor (de $120 \mathrm{~ms}$ de media frente a $80 \mathrm{~ms}$ en la rótica) y una asimetría y apuntamiento menores que en las róticas fricativas.

En este aspecto, los resultados coinciden con los reportados por Quilis y Carril (1971), que en un análisis de las vibrantes fricativas intervocálicas en diferentes zonas de América Latina comparan estas realizaciones con la sibilante [s] y concluyen también que se trata de sonidos distintos, con una distribución de la energía en frecuencias más altas para [s]. Las róticas fricativas se asemejarían más a $[\mathrm{z}]$, que ocupa una zona de frecuencias entre las róticas y [s]. En cualquier caso, según estos autores, el punto de articulación de róticas y sibilantes no es el mismo en las variantes analizadas. Las duraciones que aportan (valor medio de $122 \mathrm{~ms}$ en posición átona y $182 \mathrm{~ms}$ en tónica), sin embargo, son mucho más elevadas que las obtenidas en el presente estudio, pero hay que tener en cuenta que se trata de variantes del fonema /r/ en posición intervocálica, y no róticas en posición implosiva. Por otra parte, descripciones de índole dialectal como las de Canfield (1981), Griffin (1965) o Rodríguez Castellano (1952) mencionan similitudes e incluso confusión entre sibilantes y róticas fricativas.

En tercer lugar, y en relación con lo expuesto en el punto anterior, ha sido posible discriminar por sus características acústicas entre las róticas fricativas prepausales y las fricativas alveolares sordas, obteniendo una tasa de clasificaciones correctas superior al $94 \%$, tanto en la clasificación de los casos originales como en la validación cruzada. Esta alta tasa permite afirmar que, salvo en un porcentaje relativamente pequeño, los sonidos róticos realizados con una fricción son claramente distintos de las realizaciones del fonema fricativo /s/. Aun así, en un $6 \%$ de los casos el vector de las variables no ha sido adecuado para discriminar correctamente los sonidos, por lo que en ocasiones los locutores pueden pronunciar róticas en posición prepausal de un modo muy similar a la fricativa /s/. Podría comprobarse mediante una prueba de per- cepción si los hablantes confunden las róticas fricativas con la /s/ o, por el contrario, las discriminan de forma clara.

Las realizaciones analizadas corresponden a un contexto muy concreto (prepausal) de una variedad específica (español centropeninsular). En la introducción se han incluido referencias a otras variedades dialectales que presentan róticas fricativas o asibiladas, que podrían ser similares a las descritas aquí o diferir en gran medida. La intuición de los autores es que, al menos en el caso de las realizaciones que corresponden a la vibrante $/ \mathrm{r}$, , se trata de sonidos distintos. Es, sin embargo, una apreciación subjetiva, basada en la percepción. Sería necesario comparar acústicamente nuestros datos con los obtenidos en otros estudios en que se analizan róticas fricativas, para así determinar si existen similitudes entre ellas. Sessarego (2011) y Morgan y Sessarego (en prensa), al analizar las róticas en el español del altiplano de Bolivia, documentan un alto porcentaje de realizaciones fricativas con diferente grado de estridencia, tanto róticas como sibilantes, y aportan una caracterización acústica detallada de cada una de las categorías observadas. A pesar de que, a grandes rasgos, las realizaciones sibilantes se asemejan a las róticas fricativas descritas en el presente estudio, parece que la distribución de la energía no es la misma, y en cualquier caso, se trata allí de realizaciones del fonema /r/ y en posición intervocálica, por lo que la comparación con el contexto prepausal no sería válida. Sí resultaría interesante poder contrastar los datos acústicos con los obtenidos por Bradley (2004) para posición implosiva, pero no aparecen lo suficientemente detallados.

Finalmente, ya se ha comentado que estos sonidos presentan una alta variación inter-locutor, por lo que los valores presentados en este estudio deben considerarse como orientativos. La constatación de esta variación inter-locutor tiene implicaciones en distintos ámbitos aplicados. Por ejemplo, constituye una variable interesante a tener en cuenta para la identificación forense de locutores. Otro ejemplo tiene que ver con el reconocimiento y síntesis del habla, especialmente si se tiene interés en producir róticas prepausales acordes al uso real por parte de los locutores.

\section{AGRADECIMIENTOS}

Este trabajo ha sido parcialmente financiado por los proyectos de investigación FFI2012-34601, FFI201459848-C2-1-P y FFI2012-31415 del Ministerio de Economía y Competitividad (España).

\section{REFERENCIAS}

Argüello, F. M. (1978). The žeista dialect of Spanish spoken in Ecuador: A phonetic and phonological study (tesis doctoral). The Pennsylvania State University. 
Barreiro Bilbao, S. C. (1999-2000). Análisis acústico comparado de las fricativas castellanas no sibilantes en realizaciones aisladas. Contextos, 33-36, 243-261.

Blecua, B. (1999). Características acústicas de la vibrante múltiple del español en habla espontánea. En Actas del I Congreso de Fonética Experimental (pp. 119-126). Tarragona: Servei de Publicacions, Universitat de Barcelona y Universitat Rovira i Virgili.

Blecua, B. (2001). Las vibrantes del español: Manifestaciones acústicas y procesos fonéticos (tesis doctoral). Bellaterra: Universitat Autònoma de Barcelona. Disponible en http://hdl.handle.net/10803/4859

Blecua, B. (2006). Estudio acústico de la vibrante en posición implosiva. En Filología y lingüística. Estudios ofrecidos a Antonio Quilis (vol. 1), pp. 97-112. Madrid: CSIC, UNED y Universidad de Valladolid.

Blecua, B., Cicres, J., \& Gil, J. (en prensa). Variación en las róticas del español y su implicación en la identificación del locutor. $R e$ vista de Filología Románica, Anejo 8 (2014): Estudios de fonética y fonología románicas.

Bradley, T. G. (2001). The phonetics and phonology of rhotic duration contrast and neutralization (tesis doctoral). The Pennsylvania State University. http://dx.doi.org/doi:10.7282/T3VT1QX9

Bradley, T. G. (2004). Gestural timing and rhotic variation in Spanish codas. En T. L. Face (Ed.), Laboratory approaches to Spanish phonology (pp. 195-220). Berlin: Mouton de Gruyter.

Bradley, T. G. (en prensa). Consonantes róticas: Descripción fonética. En J. Gil \& J. Llisterri (Eds.), Fonética y fonología descriptivas de la lengua española (cap. 21). Madrid: Consejo Superior de Investigaciones Científicas. Disponible en https://www. academia.edu/7095921/Consonantes r\%C3\%B3ticas Descripci $\%$ C3\%B3n_fon $\%$ C3\%A9tica

Bradley, T. G., \& Willis, E. W. (2012). Rhotic variation and contrast in Veracruz Mexican Spanish. Estudios de Fonética Experimental, 21, 43-74.

Canfield, D. L. (1981). Spanish pronunciation in the Americas. Chicago, IL: University of Chicago Press.

Cerdà, R. (1968). Algunas observaciones en torno a la definición de «-r» española. Boletín de Filología Española, 7(26-27), 19-24.

Cicres, J. (2011). Los sonidos fricativos sordos y sus implicaciones forenses. Estudios Filológicos, 48, 33-48. http://dx.doi org/10.4067/S0071-17132011000200003

Colantoni, L. (2001). Mergers, chain shifts, and dissimilatory processes: Palatals and rhotics in Argentine Spanish (tesis doctoral). Minneapolis: University of Minnesota.

Fernández, S., Feijóo, S., Balsa, R., \& Barros, N. (1998). Acoustic cues for the auditory identification of the Spanish fricative /f/ En Proceedings of the International Conference on Spoken Language Processing (ICSLP '98), 5, 2151-2154.

Forrest, K., Weismer, G., Milenkovic, P., \& Dougall, R. N. (1988). Statistical analysis of word-initial voiceless obstruents: Preliminary data. Journal of the Acoustical Society of America, 84, 115-124. http://dx.doi.org/10.1121/1.396977

Fu, H., Rodman, R. D., McAllister, D. F., Bitzer, D. L., \& Xu, B. (1999). Classification of voiceless fricatives through spectral moments. En Proceedings of the 5th International Conference on Information Systems Analysis and Synthesis (ISAS '99), pp. 307-311. Skokie, IL: International Institute of Informatics and Systemics.

García Antuña, M. (2012). Análisis acústico-sonográfico de las consonantes róticas $(/ \mathrm{r} / y / \mathrm{r} /)$ para su aplicación en entornos de estudios forenses (trabajo de fin de máster). Alcalá de Henares: Instituto Universitario de Investigación en Ciencias Policiales. Facultad de Derecho, Universidad de Alcalá de Henares.

Gili Gaya, S. (1921). La «r» simple en la pronunciación española. Revista de Filología Española, 8, 271-280.

Griffin, D. (1965). Rotacismo y aspiración: Una nota sobre cronología dialectal. Boletín de Filología, 17, 407-411.
Henriksen, N. C., \& Willis, E. W. (2010). Acoustic characterization of phonemic trill production in Jerezano Andalusian Spanish. En M. Ortega-Llebaria (Ed.), Selected Proceedings of the 4th Conference on Laboratory Approaches to Spanish Phonology (pp. 115-127). Somerville, MA: Cascadilla Proceedings Project.

Hualde, J. I. (2005). The sounds of Spanish. Cambridge, UK: Cambridge University Press.

Jongman, A., Wayland, R., \& Wong, S. (2000). Acoustic characteristics of English fricatives. Journal of the Acoustical Society of America, 108(3), 1252-1263. http://dx.doi.org/10.1121/1.1288413

Lenz, R. (1892). Chilenische Studien. Phonetische Studien, 5 , 272-293.

Lindau, M. (1985). The story of /r/. En V. A. Fromkin (Ed.), Phonetic linguistics. Essays in honour of Peter Ladefoged (pp. 157168). Orlando, FL: Academic Press.

Lope Blanch, J. M. (1978). Una nota sobre los sonidos vibrantes. Anuario de Letras, 16, 247-250.

Malmberg, B. (1965). Estudios de fonética hispánica: Collectanea Phonetica (vol. 1). Madrid: CSIC.

Martínez Celdrán, E. (1984). Fonética. Barcelona: Teide.

Martínez Celdrán, E., \& Fernández Planas, A. M. (2007). Manual de fonética española. Articulaciones y sonidos del español. Barcelona: Ariel.

Martínez Celdrán, E., \& Rallo, L. (1995). [^-r]: ¿Dos clases de sonidos? Estudios de Fonética Experimental, 7, 181-193.

Massone, M. I. (1988). Estudio acústico y perceptivo de las consonantes nasales y líquidas del español. Estudios de Fonética Experimental, 3, 13-34

Morgan, T., \& Sessarego, S. (en prensa). A phonetic analysis of $/ \mathrm{r} /$ in Highland Bolivian Spanish. Spanish in Context, 13(2).

Navarro Tomás, T. (1918/1985). Manual de pronunciación española (22. ${ }^{\mathrm{a}}$ ed.). Madrid: CSIC.

Quilis, A. (1993). Tratado de fonología y fonética españolas. Madrid: Gredos.

Quilis, A., \& Carril, R. B. (1971). Análisis acústico de [řr] en algunas zonas de Hispanoamérica. Revista de Filología Española, 59(34), 271-316. http://dx.doi.org/10.3989/rfe.1971.v54.i3/4.844

Real Academia Española (2011). Nueva gramática de la lengua española. Vol. 3: Fonética y fonología. Madrid: Espasa.

Recasens, D. (1991). On the production characteristics of apicoalveolar taps and trills. Journal of Phonetics, 19, 267-280.

Recasens, D. (1993). Fonètica i fonologia. Barcelona: Enciclopèdia Catalana.

Recasens, D., \& Pallarès, M. D. (1999). A study of $/ \mathrm{f} /$ and $/ \mathrm{r} /$ in the light of the "DAC" coarticulation model. Journal of Phonetics, 27(2), 143-169. http://dx.doi.org/10.1006/jpho.1999.0092

Rodríguez Castellano, L. (1952). La variedad dialectal del Alto Aller. Oviedo: Instituto de Estudios Asturianos.

Romero, J. (2008). Gestural timing in the perception of Spanish $\mathrm{r}+\mathrm{C}$ clusters. En L. Colantoni \& J. Steele (Eds.), Selected Proceedings of the 3rd Conference on Laboratory Approaches to Spanish Phonology (pp. 59-71). Somerville, MA: Cascadilla Proceedings Project.

Sessarego, S. (2011). Phonetic analysis of /sr/ clusters in Cochabambino Spanish. En L. A. Ortiz-López (Ed.), Selected Proceedings of the 13th Hispanic Linguistics Symposium (pp. 251263). Somerville, MA: Cascadilla Proceedings Project.

Walsh Dickey, L. (1997). The phonology of liquids (tesis doctoral). Disponible en ProQuest (tesis n. ${ }^{\circ}$ AAI9721443).

Zahler, S., \& Daidone, D. (2014). A variationist account of trill /r/ usage in the Spanish of Málaga. IULC Working Papers: Special Vol.: Quantitative approaches to the study of sociolinguistic phenomena across Spanish varieties, 14(2), 17-42. Blomington: Indiana University Working Papers in Linguistics.

Zsiga, E. (1993). Features, gestures, and the temporal aspects of phonological organization (tesis doctoral). New Haven, CT: Yale University. 